\title{
Increased quantum efficiency and reduced red-shift in polymer nanoparticle luminophors
}

\author{
Ilkem Ozge Huyal, Tuncay Ozel, Donus Tuncel, and Hilmi Volkan Demir \\ Department of Physics, Department of Chemistry, Department of Electrical and Electronics Engineering, \\ Nanotechnology Research Center, and Institute of Materials Science and Nanotechnology, \\ Bilkent University, Ankara 06800, Turkey
}

Today a growing energy demand necessitates the development of cost-effective, energy-efficient lighting systems. For this purpose, light-emitting diodes (LEDs) provide the ability to generate cheaper, highquality white light. In the wavelength-conversion technique, different luminophors such as phosphors, nanocrystals, and polymers, integrated on pump LED platforms, are exploited to generate white light [14]. Among these materials, conducting polymers are especially promising because of their ease of processability and possible low-cost, large-area applications [1,5]. Of such $\pi$-conjugated systems, poly(9,9-dialkylfluorene)s are particularly attractive as light emitters because of their high quantum efficiency and good thermal stability [6, 7].

The use of such fluorescent polymers in their film forms is indispensable for optoelectronic device applications. This requires the solutions of $\pi$-conjugated polymers to be deposited by spin-coating or drop-casting onto solid substrates. This in turn results in electronic coupling of polymer chains (interchain coupling), which is essential for charge carrier transport in the film state. The presence of interchain coupled states induces an efficient energy transfer from higher energy intrachain singlet excitons to lower lying ones that are delocalized over several polymer chains in intimate contact with each other. Indeed, the aggregate-induced depopulation of the initially excited intrachain excitons leads to a red-shifted emission band, which is also associated with a significant reduction in the fluorescence quantum efficiency $\left(\Phi_{\mathrm{PL}}\right)$ in film state on the order of 50\% [8-12]. To address the problem of efficiency reduction in film form, we proposed and demonstrated the formation of polymer nanoparticle luminophors, leading to a significant decrease in the number of interchain species upon nanoparticle formation and yielding highly efficient luminescent nanoparticles.
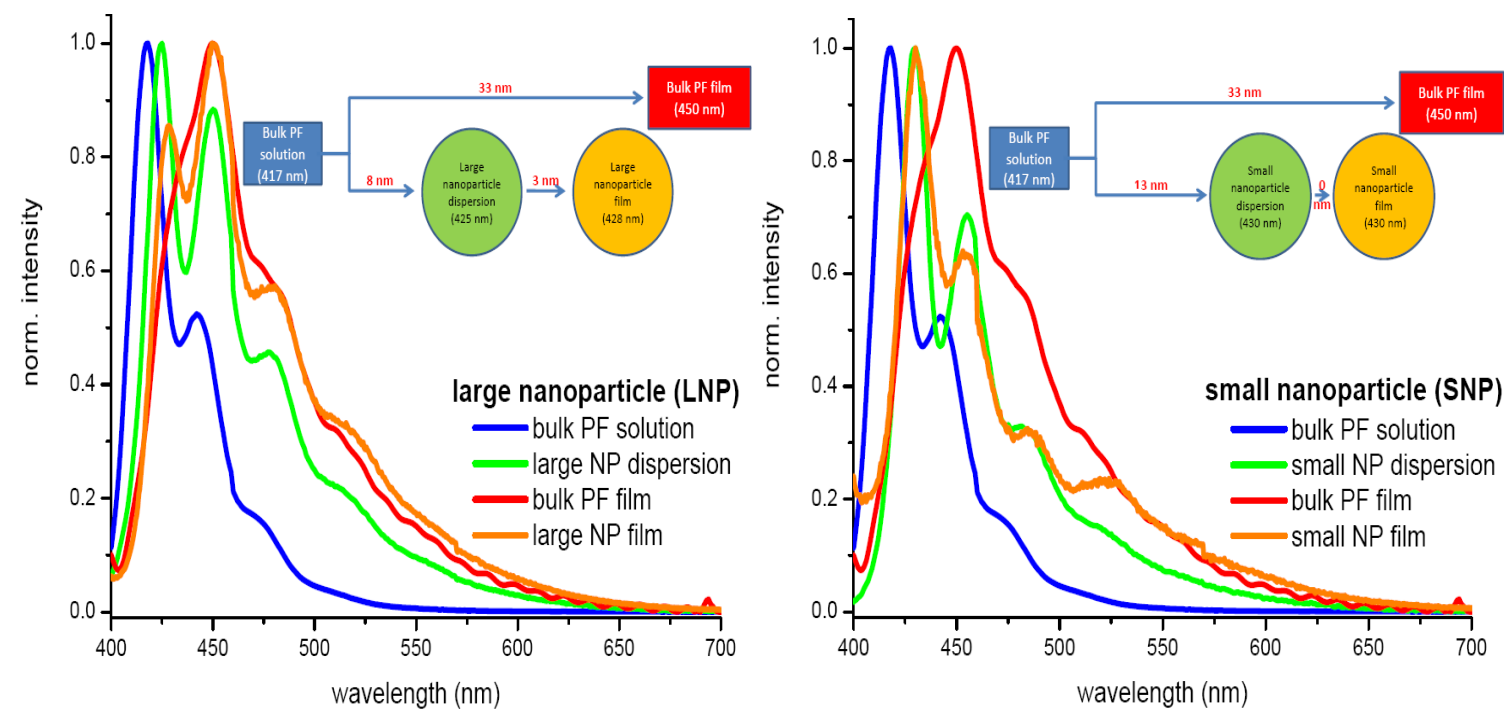

Figure 1. Observed spectral photoluminescence shifts for 0-0 electronic transition of (a) large nanoparticles (LNPs) and (b) small nanoparticles (SNPs) in their dispersion and film states relative to bulk PF in solution and thin film.

We targeted to prepare polymer nanospheres with different sizes at different concentrations to achieve higher control over the emission color and reduce loss in the quantum efficiency when the polymer is transformed into its film state. The formation of polymer nanoparticles was realized via the facile reprecipitation method in which a very dilute polymer solution, with THF as the water soluble organic solvent, was reprecipitated at different amounts into sonicated water [13-16]. Herein, we present the preparation of highly efficient polyfluorene (PF) nanoparticles ranging from 5 to $70 \mathrm{~nm}$ in diameter 
prepared by this method. In their film forms, the nanoparticles with larger size distribution exhibited $\Phi_{\mathrm{PL}}$ of $43 \%$, while smaller ones exhibited an even higher $\Phi_{\mathrm{PL}}$ of $68 \%$, which surpassed $\Phi_{\mathrm{PL}}$ of the PF thin film with values ranging from $23 \%$ to $44 \%$ depending on the film thickness.

Also, particularly interesting is that spectral shifts from dispersion to film state are greatly reduced for the nanoparticles, which is advantageous in terms of spectral control and stability. Figure 1 shows the spectral PL shifts for both large nanoparticles (LNP) and small nanoparticles (SNP). Figure 2 depicts an AFM image of these LNPs, which exhibited a size distribution of 5 to $70 \mathrm{~nm}$, while the smaller ones were observed to range from 5 to $30 \mathrm{~nm}$. As shown in Figure 1, while the peak of 0-0 electronic transition redshifted by a total of $33 \mathrm{~nm}$ going from PF bulk solution to PF bulk thin film, this red-shift was only a total of $11 \mathrm{~nm}$ going from PF bulk solution to PF large nanoparticle (LNP) film, and only $13 \mathrm{~nm}$ going from PF bulk solution to PF small nanoparticle (SNP) film.

Furthermore, we analyze energy transfer mechanisms in these polymer nanoparticles and hybrids of different polymer nanoparticles via time-resolved measurements. The formation of hybrid polymer nanoparticles is likely to be a promising method for the generation of highly efficient white light that relies on the use of at least two types of conjugated polymers assembled into novel nanostructures.

In summary, using a polyfluorene derivative, we achieved increased fluorescence quantum efficiency and reduced red-shift in the film form of polymer nanoparticle luminophors, when compared to directly spincoated polymer thin films.

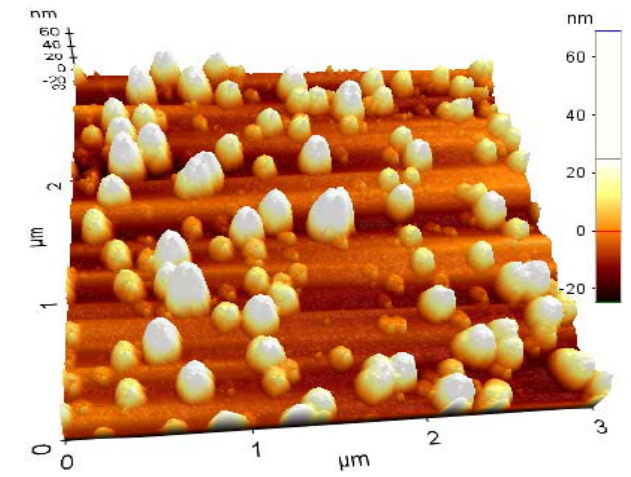

Figure 2. Atomic force microscopy (AFM) topography of PF large nanoparticles (LNPs).

Acknowledgement: This work is supported by EU-PHOREMOST NoE 511616, EU Marie Curie IRG MOON 021391, and TUBITAK 106E020, 105M027, 104E114, 107E088, 107E297, 105E065, and 105E066. Also, HVD acknowledges additional support from TUBA GEBIP and European Young Investigator (EURYI) award programs.

References

[1] G. Heliotis et al., Appl.Phys. Lett., 2005, 87, 103505.

[2] S. Nizamoglu and H. V. Demir, Nanotechnology, 2007, 18, 405702.

[3] I. O. Huyal, T. Ozel, U. Koldemir, S. Nizamoglu, D. Tuncel, and H. V. Demir, Opt. Express, 2008, 16, 1115.

[4] I. O. Huyal, U. Koldemir, T. Ozel, H. V. Demir, and D. Tuncel, J. Mater. Chem., 2008, DOI:10.1039/b802910e.

[5] F. Hide, P. Kozodoy, S. P. DenBaars, and A. Heeger, Appl. Phys. Lett., 1997, 70, 2664

[6] K. Hosoi, T. Mori, T. Mizutani, T. Yamamoto, and N. Kitamura, Thin Sol. Films, 2003, 438-439.

[7] H. Lambeth, Electroluminescence of Conjugated Aromatic Polymers in Organic Light Emitting Diodes (2004).

[8] S. C. J. Meskers, J. Hübner, M. Oestreich, H. Bassler, Chem. Phys. Lett., 2001, 339, 223.

[9] A. Haugeneder, U. Lemmer, U. Scherf, Chem. Phys. Lett., 2002, 351, 354.

[10] J. Gierschner, H.-J. Egelhaaf, D. Oelkrug, and K. Müllen, J. Fluorescence, 1998, 8, 37.

[11] I. B. Martini, A. D. Smith, and B. J. Schwartz, Phys. Rev. B, 2004, 69, 035204.

[12] F. B. Dias, A. L. Macanita, J. S. de Melo, and H. D. Burrows, J. Chem. Phys., 2003, 118, 7119.

[13] C. Syzmanski, C. Wu, J. Hooper, M. A. Salazar, A. Perdomo, A. Dukes, and J. McNeill, J. Phys. Chem B, 2005, $109,8543$.

[14] F. Kong, X. L. Wu, G. S. Huang, R. K. Yuan, P. K. Chu, Thin Sol. Films doi:10.1016/j.tsf.2007.12.055 (2008).

[15] F. Wang, M.-Y. Han, K. Y. Mya, Y. Wang, and Y.-H. Lai, J. Am. Chem. Soc., 2005, 127, 10350.

[16] N. Kurokawa, H. Yoshikawa, N. Hirota, K. Hyodo, and H. Masuhara, ChemPhysChem, 2004, 5, 1609. 